were genotyped in 386 Caucasian IgAV patients (the largest series of Caucasian patients with IgAV ever assessed for genetic studies) and 806 sex and ethnically matched healthy controls by TaqMan assays.

Results: No statistically significant differences in the genotype and allele frequencies between patients with IgAV and healthy controls were observed when each genetic variant of BAFF APRIL and BAFFR was analyzed independently (Table 1). Likewise, no statistically significant differences in genotype and allele frequencies of $B A F F A P R I L$ or BAFFR were found when patients with IgAV were stratified according to the age at disease onset or to the presence/absence of gastrointestinal or renal manifestations. Similar results were disclosed when haplotype frequencies of $A P R I L$ and BAFFR were compared between patients with IgAV and healthy controls as well as patients with IgAV stratified according to the age at disease onset or to the presence/absence of gastrointestinal or renal manifestations.

Conclusion: Our results suggest that the BAFF-APRIL-BAFFR pathway does not contribute to the genetic network underlying IgAV.

REFERENCES:

[1] J Exp Med 1999;190:1697-710; [2] Science 1999;285:260-3; [3] Nat Genet 2005;37:829-34; [4] Nat Immunol 2002;3:822-9; [5] N Engl J Med 2017;376:1615-26; [6] N Engl J Med 2013;368:2402-14; [7] Autoimmun Rev 2018;17:301-315.

Table 1. Genotype and allele frequencies of BAFF, APRIL and BAFFR genes in patients with IgA vasculitis and healthy controls.

\begin{tabular}{|c|c|c|c|c|c|c|c|c|}
\hline Polymorphism & Locus & $1 / 2$ & Data set & $1 / 1$ & $1 / 2$ & $2 / 2$ & 1 & 2 \\
\hline \multirow[t]{2}{*}{ rs374039502 } & BAFF & T/A & Patients & 91.9 (353) & $8.1(31)$ & 0 & $95.9(737)$ & $4.1(31)$ \\
\hline & & & Controls & 91.5 (733) & $8.1(65)$ & $0.4(3)$ & 95.6 (1531) & $4.4(71)$ \\
\hline \multirow[t]{2}{*}{ rs11552708 } & APRIL & $G / A$ & Patients & 78.1 (299) & $20.6(79)$ & $1.3(5)$ & $88.4(677)$ & $11.6(89)$ \\
\hline & & & Controls & 77.9 (625) & $20.4(164$ & $1.6(13)$ & 88.1 & 11.9 (190) \\
\hline \multirow[t]{2}{*}{ rs6608 } & APRIL & $\mathrm{C} / \mathrm{T}$ & Patients & 71.9 (277) & $26.0(100)$ & $2.1(8)$ & 84.9 & $15.1(116)$ \\
\hline & & & Cont & 70.0 & $27.6(221)$ & $2.5(20)$ & 83.7 (1343) & 16.3 \\
\hline \multirow[t]{2}{*}{ rs7290134 } & BAFFR & $A / G$ & Patients & $58.0(224)$ & $36.3(140)$ & $5.7(22)$ & $76.2(588)$ & $23.8(184)$ \\
\hline & & & Controls & $57.2(459)$ & $36.4(292)$ & $6.5(52)$ & $75.3(1210)$ & 24.6 (396) \\
\hline \multirow[t]{2}{*}{ rs77874543 } & BAFFR & $\mathrm{G} / \mathrm{C}$ & Patients & $82.7(316)$ & $16.0(61)$ & $1.3(5)$ & 90.7 (693) & $9.3(71)$ \\
\hline & & & Controls & $83.0(666)$ & 16.6 (133) & $0.4(3)$ & $91.3(1465)$ & 8.7 (139) \\
\hline
\end{tabular}

Acknowledgements: This study was supported by European Union FEDER funds and "Fondo de Investigaciones Sanitarias" (grant PI18/00042) from 'Instituto de Salud Carlos III' (ISCIII, Health Ministry, Spain). DP-P is a recipient of a Río Hortega programme fellowship from the ISCIII, co-funded by the European Social Fund (ESF, 'Investing in your future') [grant number CM20/00006]; SR-M is supported by funds of the RETICS Program co-funded by the European Regional Development Fund (ERDF) [grant number RD16/0012/0009]; VP-C is supported by a pre-doctoral grant from IDIVAL [grant number PREVAL 18/01]; BA-M is a recipient of a 'López Albo' Post-Residency Programme funded by Servicio Cántabro de Salud; LL-G is supported by funds of IDIVAL [grant number INNVAL20/06]; RL-M is a recipient of a Miguel Servet type I programme fellowship from the ISCIII, co-funded by the European Social Fund (ESF, 'Investing in your future') [grant number CP16/00033].

Disclosure of Interests: Diana Prieto-Peña Speakers bureau: Abbvie, Pfizer, Roche, Bristol-Myers, Janssen and MSD, Consultant of: Abbvie, Pfizer, Roche, Bristol-Myers, Janssen and MSD, Grant/research support from: Abbvie, MSD and Roche, Sara Remuzgo Martinez: None declared, Fernanda Genre: None declared, Verónica Pulito-Cueto: None declared, Belén Atienza-Mateo: None declared, Belén Sevilla: None declared, Javier Llorca: None declared, Norberto Ortego: None declared, Leticia Lera-Gómez: None declared, Maite Leonardo: None declared, Ana Peñalba: None declared, J. Narváez: None declared, Luis Martín-Penagos: None declared, Jose Alberto Miranda-Filloy: None declared, LUIS CAMINAL MONTERO: None declared, PAZ COLLADO: None declared, Javier Sanchez Perez: None declared, Diego de Argila: None declared, Esteban Rubio-Romero: None declared, MANUEL LEON LUQUE: None declared, Juan María Blanco-Madrigal: None declared, E. Galindez: None declared, Javier Martin Ibanez: None declared, Santos Castañeda: None declared, Ricardo Blanco Speakers bureau: Abbvie, Pfizer, Roche, Bristol-Myers, Janssen and MSD, Consultant of: Abbvie, Pfizer, Roche, Bristol-Myers, Janssen and MSD, Grant/ research support from: Abbvie, MSD and Roche, Miguel A González-Gay Speakers bureau: Pfizer, Abbvie, MSD, Grant/research support from: Pfizer, Abbvie, MSD, Raquel López-Mejías: None declared DOI: 10.1136/annrheumdis-2021-eular.707

\section{POS0114 ABERRANT MONOCYTE SUBSETS IN PATIENTS WITH BEHÇET'S DISEASE}

C. $\mathrm{Li}^{1,2}$, J. Liu ${ }^{1}$, H. Chen ${ }^{1}$, W. Zheng ${ }^{1}{ }^{1}$ Peking Union Medical College Hospital, Rheumatology and Clinical Immunology, Beijing, China; ${ }^{2}$ Peking University Shougang Hospital, Rheumatology, Beijing, China
Background: Behçet's Disease (BD) is a systemic vasculitis of unknown etiology[1]. Monocytes closely related to inflammation potentially contribute to BD's pathogenesis. They are classified into three subsets: classical monocytes (CM) intermediate monocytes (IM) and non-classical monocytes (NCM). Abnormalities of monocyte subsets have been reported in many infectious, inflammatory and autoimmune diseases[2-6], but their implication in BD remains elusive.

Objectives: To investigate the distribution, phenotypes and functions of monocyte subsets in BD and explore their roles in BD pathogenesis.

Methods: The frequencies and phenotypes of monocyte subsets in $\mathrm{BD}$ and healthy controls ( $\mathrm{HC}$ ) were determined by flow cytometry, and their correlation with clinical parameters was analyzed. Intracellular cytokines and phosphorylated signal proteins [phosphorylated p65(p-p65) and phosphorylated p38(p-p38)] were determined in LPS-activated monocyte subsets by flow cytometry. Monocyte subsets of BD and $\mathrm{HC}$ were sorted and co-cultured with naïve CD4 + T cells, and Th1 cell frequencies were measured on day 5.

Results: A higher IM $(9.0 \pm 3.6 \%$ vs. $4.5 \pm 2.0 \%, \mathrm{p}<0.01)$ and lower NCM $(2.6 \pm 1.2 \%$ vs. $4.2 \pm 2.0 \%, p<0.01$ ) population in $B D$ patients were noted. BD IM were positively correlated with CRP $(r=0.5456, p<0.05)$, ESR $(r=0.4683, p=0.05)$, and the serum level of TNF-a $(r=0.7372, p<0.001)$ and IL- $6(r=0.5013, p<0.05)$. BD NCM were negatively correlated with CRP $(r=0.4822, p<0.05)$ and the serum level of IgM $(r=-0.7830, p<0.001)$. Moreover, BD IM decreased $(12.3 \pm 3.8 \%$ vs. $5.7 \pm 3.6 \%, p<0.05)$, while BD NCM increased $(2.6 \pm 1.3 \%$ vs. $3.5 \pm 1.5 \%$, $\mathrm{p}<0.01)$ after BD patients achieved remission. CD11b and CD64 expression on $\mathrm{CM}$, IM and NCM in BD were enhanced. BD CM promoted TNF-a $(61.0 \pm 11.4 \%$ vs $48.3 \pm 9.9 \%, p<0.05)$ and IL-6 $(7.2 \pm 5.4 \%$ vs $1.9 \pm 1.7 \%, p<0.05)$ production and facilitated Th1 differentiation. BD IM promoted IL-6 production $(6.2 \pm 3.8 \%$ vs $2.6 \pm 1.6 \%, p<0.05)$. Furthermore, we demonstrated a higher level of $p-p 65$ $(12.8 \pm 2.9 \%$ vs $3.3 \pm 1.1 \%, p<0.01)$ in $\mathrm{BD}$ CM and increased $\mathrm{p}-\mathrm{p} 65(3.2 \pm 0.6 \%$ vs $0.01 \pm 0.01 \%, p<0.01)$ and p-p38 $(1.1 \pm 0.6 \%$ vs $0.03 \pm 0.01 \%, p<0.01)$ in BD IM.

Conclusion: To our knowledge, our study is the first study on monocyte subsets in $\mathrm{BD}$. Our data highlighted the aberrant populations of IM and $\mathrm{CM}$ in $\mathrm{BD}$, potentially implicated in BD pathogenesis.

\section{REFERENCES:}

[1] Ahmet, Gül, Pathogenesis of Behçet's disease: autoinflammatory features and beyond, Semin. Immunopathol. 37 (2015) 413-418.

[2] G.Fingerle, A.Pforte, B.Passlick, et al.The novel subset of CD14+/CD16+ blood monocytes is expanded in sepsis patients, Blood. 82 (1993) 3170-3176.

[3] M.Moniuszko, A. Bodzenta-Lukaszyk, K. Kowal, et al. Enhanced frequencies of CD14++CD16+, but not CD14+CD16+, peripheral blood monocytes in severe asthmatic patients, Clin. Immunol. 130 (2009) 338-346.

[4] M.D.Sanchez, Y.Garcia, C.Montes,et al.Functional and phenotypic changes in monocytes from patients with tuberculosis are reversed with treatment, Microbes. Infec. 8 (2006) 2492-2500.

[5] S.Koch, T.Kucharzik, J.Heidemann,et al. Investigating the role of proinflammatory CD16+ monocytes in the pathogenesis of inflammatory bowel disease, Clin. Exp. Immunol. 161 (2010) 332-341.

[6] H. Zhu, F. Hu, X. Sun,et al.CD16(+) Monocyte Subset Was Enriched and Functionally Exacerbated in Driving T-Cell Activation and B-Cell Response in Systemic Lupus Erythematosus, Front. immunol. 7 (2016) 512.

Disclosure of Interests: None declared

DOI: 10.1136/annrheumdis-2021-eular. 1157

\section{POS0115 \\ RISK FACTORS FOR CORONARY HEART DISEASE AND ATHEROSCLEROSIS IN PATIENTS WITH BECHET'S DISEASE}

R. Goloeva' 1 , Z. Alekberova', T. Popkova', S. Glukhova ${ }^{1}$. $V$ A Nasonova Research Institute of Rheumatology, systemic rheumatic diseases, Moscow, Russian Federation

\section{Background:}

Objectives: To evaluate traditional risk factors for clinically manifest and asymptomatic atherosclerosis in pts with BD.

Methods: Traditional risk factors for atherosclerosis were evaluated in 95 pts with Bechet's disease (BD) and 40 healthy age and gender matched controls. The male-to-female ratio was 3,7:1, pts' mean age was 29.7 (23-35) y, the mean age at the disease onset - 19,9 (14-25) y, and mean disease duration - 9,6 (4-15) y. The common carotid artery intima-media thickness (IMT) was evaluated by high resolution B-mode ultrasonography. A plaque was defined as a local intima-media thickening $\geq 1,2 \mathrm{~mm}$, with reference IMT thickness $<0,9 \mathrm{~mm}$. Serum concentration of high-sensitivity C-reactive protein (hs CRP) was measured by immunonephelometric assay (BN-100 Analyzer; Dade Behring). Lipid profile evaluation included total cholesterol, TGs, HDL, LDL and atherogenic index. Results: Traditional risk factors for atherosclerosis (one or more) were found in $85,3 \%$ of BD pts, dyslipidemia was the most common risk factor and was present in $76,84 \%$ of pts, mostly due to increased total cholesterol (TC) and 
low-density lipoprotein cholesterol (LDL-C) levels. 22,1\% had arterial hypertension $(\mathrm{AH}), 29,5 \%$ were current smokers, $27,4 \%$ had excess body weight, $3,1 \%$ had family history of cardiovascular diseases (CVD). Traditional risk factors for atherosclerosis were found in $80 \%$ out of 40 gender and age matching subjects from the control group, i.e., showing practically the same prevalence as in BD pts.

Table 1. The incidence-rate of traditional risk factors in BD patients and controls

\begin{tabular}{lccc}
\hline Variables & BD pts $(\mathrm{n}=95)$ & Controls $(\mathrm{n}=40)$ & $\mathrm{p}$ \\
\hline Age, years & $29,0[23,0 ; 35,0]$ & $29,0[25,0 ; 32,0]$ & $\mathrm{NS}$ \\
$\mathrm{BMI}, \mathrm{kg} / \mathrm{m}^{2}$ & $23,1[21,1 ; 25,5]$ & $23,8[22,0 ; 26,0]$ & $\mathrm{NS}$ \\
$\mathrm{BMI} \geq 25 \mathrm{~kg} / \mathrm{m}^{2}, \mathrm{n}(\%)$ & $26(27,4)$ & $15(37,5)$ & $\mathrm{NS}$ \\
Arterial hypertension, $\mathrm{n}(\%)$ & $21(22,1)$ & $4(10)$ & $\mathrm{NS}$ \\
Family history of CVD & $3(3,1)$ & $5(12,5)$ & 0,05 \\
Cigarette smoking, $\mathrm{n}(\%)$ & $28(29,5)$ & $12(30,0)$ & $\mathrm{NS}$ \\
Dyslipidemia, $\mathrm{n}(\%)$ & $73(76,8)$ & $27(67,5)$ & $\mathrm{NS}$ \\
Total cholesterol, mml/l & $5,0[4,3 ; 6,1]$ & $5,1[4,5 ; 5,7]$ & $\mathrm{NS}$ \\
Cholesterol $>5,0 \mathrm{mml} / \mathrm{l}, \mathrm{n}(\%)$ & $47(49,5)$ & $25(62,5)$ & $\mathrm{NS}$ \\
TGs, mml/l & $0,92[0,7 ; 1,3]$ & $0,89[0,60 ; 1,08]$ & $\mathrm{NS}$ \\
TGs $>1,7 \mathrm{mml} / \mathrm{l}, \mathrm{n}(\%)$ & $8(8,4)$ & $3(7,5)$ & $\mathrm{NS}$ \\
LDL, mml/l & $3,36(2,8 ; 4,0)$ & $3,3[2,7 ; 3,7]$ & $\mathrm{NS}$ \\
LDL $>3 \mathrm{mml} / \mathrm{l}, \mathrm{n}(\%)$ & $63(66,3)$ & $25(62,5)$ & $\mathrm{NS}$ \\
$\mathrm{HDL}, \mathrm{mml} / \mathrm{l}$ & $1,19[1,0 ; 1,5]$ & $1,35[1,2 ; 1,6]$ & 0,06 \\
$\mathrm{HDL} \leq 1 \mathrm{mml} / \mathrm{l}(\mathrm{male}), \leq 1,2 \mathrm{mml} / \mathrm{l}$ (female) $\mathrm{n}(\%)$ & $30(31,6)$ & $5(12,5)$ & 0,03 \\
\hline
\end{tabular}

Reduced HDL levels were more common in BD pts vs the controls - $30(31,6 \%)$ vs $5(12,5), p=0,03$ as shown in table 1 . Other traditional risk factors were similarly present in both groups with no significant difference between BD pts and the controls.

Analysis showed similar incidence of CV events (nonfatal myocardial infarction, angina pectoris and stroke) in both groups. There were no differences between BD pts and healthy people in terms of lifetime risk for CVD. High lifetime risk $\geq$ $20 \%$ was found in 4 BD pts vs 0 in the control group; moderate lifetime risk $>10 \%$ $<20 \%$ - in $6,3 \%$ of BD pts vs $2,5 \%$ in the control group, and low lifetime risk < $10 \%$-was found in $89,5 \%$ of BD pts vs $97,5 \%$ of the controls.

Average IMT values were significantly higher in BD pts $-0.68[0.60 ; 0.73]$ vs 0.63 [0.56;0.65], $p=0.008$, but at the same time, IMT thinning (up to $<0.5 \mathrm{~mm}$ ) was significantly more common in BD pts $-32.6 \%$ vs $12.5 \%$ in the control group $(p=0.01)$. HsCRP serum levels were significantly higher in BD pts $(2.42[0.5 ; 8.8]$ $\mathrm{mg} / \mathrm{I})$ than in the control group $(0.37[0.15 ; 0.75]), p=0.001$. There was a correlation between hs-CRP and BMI in BD pts $(r=0.2, p<0.05)$, but no correlation was found between hsCRP and BD activity/BD clinical manifestations.

Conclusion: Decreased HDL cholesterol levels were more common in BD patients than in the control group, just as thinning of IMT, most likely because of vasculitis.

Disclosure of Interests: None declared

DOI: 10.1136/annrheumdis-2021-eular.3431

\section{POS0116 HEART RATE VARIABILITY IN PATIENTS WITH BEHCETS DISEASE}

R. Goloeva ${ }^{1}$, Z. Alekberova ${ }^{1}$, T. Popkova ${ }^{1}$, S. Glukhova ${ }^{1}$, D. Novikova ${ }^{1}$. ${ }^{1} V$ A Nasonova Research Institute of Rheumatology, Systemic Rheumatic Diseases, Moscow, Russian Federation

Background: Behcet's disease (BD) is a systemic vasculitis affecting all types and sizes of blood vessels. Heart rate variability (HRV) reflects sympathetic -parasympathetic imbalance in the autonomic NS regulation. Low HRV values are known as independent risk factor of death and non-fatal cardiovascular events in both - survivors of a myocardial infarction and in asymptomatic population. Objectives: The aim of this study is to evaluate HRV in BD pts vs healthy controls.

Methods: The study group included $74 \mathrm{BD}$ pts (53males/21females) with disease duration of $9,0(5,0 ; 15,0) / 9,0(7 ; 20)$ years, and the control group - 32/15 age-matched healthy $\mathrm{m} / \mathrm{f}$. The following HRV parameters from $24 \mathrm{~h}$ ECG ambulatory recording were assessed: MeanNN and time-domain variables, adjusted by MeanNN (SDNNn\%, SDNNin\%, RMSSDn\%). Additionally, all traditional cardiovascular risk factors such as systolic blood pressure (SPB), smoking status, BMI values, dyslipidemia profile, ultrasonographic values of carotid intima-media thickness (IMT), and levels high sensitive CRP (hsCRP) as a marker of inflammation were evaluated.

Results: In BD patients HRV values (RMSSDn\%) were significantly lower compared to healthy controls (table 1 ).
Table 1. HRV parameters in BD patients and control group

\begin{tabular}{lcccc}
\hline Parameters & \multicolumn{2}{c}{ Males } & \multicolumn{2}{c}{ Females } \\
\hline & BD $(n=53)$ & Control $(n=32)$ & BD $(n=21)$ & Control $(n=15)$ \\
\hline Age, years & $30(24 ; 36)$ & $30(26 ; 35)$ & $32(26 ; 37)$ & $28(24 ; 31)$ \\
MeanNN, ms & $810(732 ; 849)$ & $782(732 ; 835)$ & $776(708 ; 830)$ & $764(694 ; 832)$ \\
SDNN n (\%) & $16,9(13,6 ; 19,4)$ & $17,2(16,3 ; 21,1)$ & $13,1(11,3 ; 5,3)$ & $12,2(10,7 ; 14,6)$ \\
SDNNi n (\%) & $6,8(5,1 ; 8,1)$ & $6,8(5,0 ; 8,3)$ & $7,1(6,1 ; 7,7)$ & $5,2(4,9 ; 5,7)$ \\
RMSSD n (\%) & $2,1(1,5 ; 2,3)^{\star *}$ & $4,1(2,7 ; 5,2)^{\star *}$ & $1,7(1,4 ; 3,7)^{\star}$ & $2,8(2,2 ; 3,9)^{\star}$ \\
\end{tabular}

Data are presented in median values and interquartile range, ${ }^{*} \mathrm{p}<0,05,{ }^{* *} \mathrm{p}<0,005$ vs controls.

There was a significant negative correlation in BD patients between HRV (SDNNin $\%)$ and age $(r=-0,4 ; p=0,00)$, disease duration $(r=-0,3 ; p=0,00)$, BMI $(r=$ $-0,2 ; p<0,01)$, cholesterol levels $(r=-0,3 ; p=0,00)$, LDLP $(r=-0,3 ; p=0,00)$ and increased IMT ( $\mathrm{r}=-0,2 ; p=0,04)$, and also between HRV (RMSSD\%) and age $(r=-0,2 ; p=0,04)$, disease duration $(r=-0,2 ; p=0,01)$, cholesterol levels $(r=-0,3$; $p=0,00)$, HDLP $(r=-0,2 ; p=0,04)$; a positive correlation was established between HRV (SNNN\%) and smoking $(r=-0,2 ; p=0,04)$. The control group showed positive correlation between HRV (SNNN\%) and increased IMT ( $r=0,4 ; p=0,01)$. Conclusion: HRV reduction reflects impaired sympathetic -parasympathetic regulation in $\mathrm{BD}$ pts, associated with pts' age, disease duration and presence of traditional cardiovascular risk factors: BMI, increased cholesterol levels, LDLP, and such asymptomatic manifestation of atherosclerosis as increased IMT.

Disclosure of Interests: None declared

DOI: 10.1136/annrheumdis-2021-eular.3682

\section{POS0117 RENAL AND OVERALL OUTCOMES OF DOUBLE- POSITIVE (ANCA AND ANTI-GBM ANTIBODIES) PATIENTS COMPARED TO ANCA-ASSOCIATED VASCULITIS PATIENTS WITH SEVERE RENAL INVOLVEMENT: A MULTICENTER RETROSPECTIVE STUDY WITH SYSTEMATIC RENAL PATHOLOGY ANALYSIS}

M. Clerte ${ }^{1}$, R. Philip ${ }^{2}$, C. Levi ${ }^{1}$, E. Cornec-Legall ${ }^{3}$, V. Audard ${ }^{4}$, A. Huart ${ }^{5}$, X. Puéchal ${ }^{6}$, M. Touzot ${ }^{7}$, N. Rabot ${ }^{8}$, E. Thervet ${ }^{1}$, A. Aouba ${ }^{2}$, A. Karras ${ }^{1}{ }^{1}{ }^{1}$ Division of Nephrology, University Paris Descartes, Georges Pompidou European Hospital, APHP, 75, Paris, France; ${ }^{2}$ Division of Clinical Immunology and Internal Medicine, Normandie University, UNICAEN, CHU of Caen Normandie, 14, Caen France; ${ }^{3}$ Division of Nephrology, Hospital of the Cavale Blanche, CHRU of Brest, 29, Brest, France; ${ }^{4}$ Department of Nephrology and Renal Transplantation, Reference Center-Idiopathic Nephrotic Syndrome, Henri-Mondor Hospital, APHP F-94000 Créteil, INSERM U955, Paris East Créteil University F-94000, 94, Créteil, France; ${ }^{5}$ Division of Nephrology and Transplantation, Reference Center of Rare Renal Diseases, University Paul Sabatier - Toulouse III, Hôpital Rangueil, 31, Toulouse, France; ${ }^{6}$ Division of Clinical Immunology and Internal Medecine, University Paris Descartes, Cochin Hospital, APHP, 75014, 75, Paris, France; ${ }^{7}$ Division of Dialysis and Therapeutic Apheresis, Health Center of Aura Paris Plaisance, 75, Paris, France; ${ }^{8}$ Division of Immunology and Nephrology, University François Rabelais, CHRU of Tours, 37, Tours, France

Background: Among small vessel vasculitis, double-positive patients (DPP) combining serum and/or histologic findings for glomerular basement membrane (GBM) disease, and anti-neutrophil cytoplasmic antibodies (ANCA), is a rare newly and poorly described condition.

Objectives: We aimed to compare characteristics between DPP and ANCA-associated vasculitis patients (AAVP) with severe-renal-involvement.

Methods: Retrospective multicenter study comparing 33 DPP and 45 severe-renal-involvement (serum creatinine $>300 \mu \mathrm{mol} / \mathrm{L}$ ) AAVP, all with biopsy-proven nephropathy.

Results: Except for 2 patients (6\%) who had pure renal presentation during their entire follow-up period, others exhibited at least one extrarenal manifestation: pulmonary involvement (64\%), weight loss (39\%), gastrointestinal involvement (33\%), ENT manifestations (21\%), musculoskeletal symptoms $(21 \%)$, fever (18\%), neurological (12\%), cutaneous $(6 \%)$ and/or cardiac $(6 \%)$ signs. All DPP (including up to $18 \%$ exhibiting negative serum anti-GBM antibodies) presented severe acute kidney failure with histologic GBM involvement. Compared to our AAVP, they had higher serum creatinine $(719$ versus $501 \mu \mathrm{mol} / \mathrm{L} ; \mathrm{p}=0.006)$ and a higher of patients requiring initial renal replacement therapy ( $82 \%$ vs $36 \%$; $p<0.001)$. Berden classification significantly differed $(p=0.003)$, with more crescentic glomerulonephritis and fewer sclerotic lesions in DPP. One-year renal survival was significantly lower in DPP than in AAVP $(27 \%$ versus $64 \% ; p<0.0002)$. With comparable proportions of ANCA subtypes (2/3 with anti-MPO autoantibodies), 